LAB.UFR-HEP/0009

\title{
Explicit Derivation of Yang-Mills Self-Dual Solutions on non-Commutative Harmonic Space
}

\author{
A. Belhaj ${ }^{1}$, M. Hssaini ${ }^{1}$, E.M. Sahraoui ${ }^{1,2}$, E.H. Saidi ${ }^{1}$ \\ ${ }^{1}$ High Energy Physics Laboratory, Faculty of Sciences, Rabat, Morocco. \\ 2 Sektion Physik, Universität München, Theresienstraße 3\%, 80333 München, \\ Germany. \\ sahraoui@theorie.physik. uni-muenchen.de \\ H-saidi@fsr.ac.ma
}

\begin{abstract}
We develop the noncommutative harmonic space (NHS) analysis to study the problem of solving the non-linear constraints eqs of noncommutative Yang-Mills selfduality in four-dimensions. We show that this space, denoted also as $\operatorname{NHS}(\eta, \theta)$, has two $\mathrm{SU}(2)$ isovector deformations $\eta^{(i j)}$ and $\theta^{(i j)}$ parametrising respectively two noncommutative harmonic subspaces $\operatorname{NHS}(\eta, 0)$ and $\operatorname{NHS}(0, \theta)$ used to study the selfdual and anti self-dual noncommutative Yang-Mills solutions. We reformulate the Yang-Mills self-dual constraint eqs on $\operatorname{NHS}(\eta, 0)$ by extending the idea of harmonic analyticity to linearize them. Then we give a perturbative self dual solution recovering the ordinary one. Finally we present the explicit computation of an exact self-dual solution.
\end{abstract}

July, 2000 


\section{Introduction}

Yang-Mills instantons on noncommutative geometry have been subject to some interest nowadays in connection with the recent developments in superstrings theory [1] and compactification of matrix model of M-theory [2, 3, 4]. Noncommutative instantons are involved in the study of $\mathrm{D}(\mathrm{p}-4) / \mathrm{Dp}$ brane systems $(\mathrm{p} \geq 3)$ of superstrings; in particular in the ADHM construction of the D0/D4 system [5, 6, 7] and in the determination of the vacuum solutions of the Higgs branch of supersymmetric gauge theories with eight supercharges [8, 9, 10].

In string theory, noncommutative geometry appears from the study of quantum properties of D-branes coupled to the closed string background fields $g_{\mu \nu}$ and $B_{\mu \nu}$. The boundary conditions of open strings of D-branes interpolate between Neumann and Dirichlet conditions and depend on the value of the NS-NS $B_{\mu \nu}$ field [11, 12. For large values of B (or equivalently B constant if one uses the Seiberg-Witten limit taking the closed string metric $g_{\mu \nu}$ to zero) the action of the system is dominated by its boundary terms describing a boundary world-sheet conformal field theory. In this case the correlation functions of the one dimensional boundary string fields $x^{M}(t)$ satisfy nontrivial commutation relations leading to a noncommutative spacetime. This noncommutative space has a canonical geometry extending the usual phase space geometry of quantum mechanics [13, 14]. The coordinates $x^{M}(t)$ do no longer commute and the usual product of functions is replaced by the star product of Moyal Bracket [15]. Soon after this development several studies have been devoted to develop the quantum field theory on noncommutative spaces [16, 17] and many partial results have been obtained [18, 19, 20].

The aim of this paper is to contribute to these efforts by considering the problem of noncommutative instantons in harmonic space (NHS). Our main motivations for this are: (1) harmonic space is a natural space where the problem of solving the selfdual Yang-Mills constraints may be done in straightforward way due to the important role played by harmonic analyticity discovered by Galperin et al in the mid-eighties. This latter has known many successful applications as in the off-shell formulation of extended supersymmetric and supergravity theories [21, 22, 23], in hyperKahler metrics building [24, 25, 26] and in the study of Yang-Mills self-dual solutions [27, 28. (2) Standard noncommutative instantons analysis shows that the self-dual constraint eqs are non-linear and hence difficult to solve exactly [何. The known non trivial solutions are obtained by perturbative analysis around the ordinary geometry. But here we will develop a different method based on a noncommutative harmonic space leading to non perturbative explicit solutions. We will see that NHS method offers a powerful manner to go beyond the standard perturbative solutions.

The presentation of this paper is as follows. In section 2, we review the main lines of the problem of solving self-dual Yang-Mills constraints eqs in ordinary HS. In 
section 3, we study the Yang-Mills instantons on NHS. We first construct the NHS spaces and show the existence of two subspaces $\operatorname{NHS}(\eta, 0)$ and $\operatorname{NHS}(0, \theta)$ depending on the values of the deformation parameters $\theta$ and $\eta$. Then we establish the connection between these noncommutative geometries and superstrings theory in presence of background fields $g_{\mu \nu}$ and $B_{\mu \nu}$. Next we focus our attention on $\operatorname{NHS}(\eta, 0)$ and study the perturbative solutions of the self-dual constraint eqs while the derivation of the exact solutions of these constraints is given in section 4 . Finally, section 5 will be devoted to the conclusion.

\section{Self-Dual Yang-Mills Constraints}

As promised in the introduction, we review here briefly the main lines of one of possible resolution ways of the problem of finding self-dual Yang-Mills (YM) solutions in ordinary $\mathbf{R}^{4}$. The method we will present is a powerful way exhibiting manifestly the $\mathrm{SU}(2)$ symmetry rotating the three Kahler structures of the hyperKahler moduli space of instantons. It allows in addition to reformulate the self-dual YM constraint eqs as integrability conditions for the existence of analytic homeomorphisms of patches of $\mathbf{C}^{2} \sim \mathbf{R}^{4}$. This method permits also to construct explicit solutions of the self-dual constraints by working in harmonic space based on first realising geometrically the $\mathrm{SU}(2) \simeq \mathbf{S}^{3}$ symmetry of the instanton hyperKahler moduli space and second using the concept of harmonic analyticity extending the usual complex analyticity where only one Kahler structure is manifestly exhibited.

To fix the ideas, we will start by describing the fundamentals of harmonic analyticity for YM instantons. Recall that such analyticity was successfully exploited in different occasions. In particular in the off-shell superspace formulation of supersymmetric field theories with eight supercharges [21, 29, 24], $d=4 \mathcal{N}=2$ and $d=2$ $\mathcal{N}=4$ (conformal) supergravity theories [23, 30, 31, 32] and in the hyperKahler metrics building [24, 25, 26, 33.

\subsection{Harmonic Analyticity}

Since the analysis we will present hereafter is valid for generalized self-dual YM fields in ordinary $\mathbf{R}^{4 n}, n=1,2, \ldots$, we shall give the machinery for $\mathbf{R}^{4 n}$ and consider the particular leading case whenever it is necessary. Roughly speaking $\mathbf{R}^{4 n}$ is a real commutative Euclidean flat space whose local coordinates $\left\{x^{M}, M=1, \ldots, 4 n\right\}$ obey generally the following natural identities

$$
\begin{array}{cl}
\overline{x^{M}} & =x^{M} \\
x^{M} x^{N}-x^{N} x^{M} & =0
\end{array}
$$




$$
x^{M} \rightarrow x^{M} \quad=\Lambda_{N}^{M} x^{N}
$$

which define the reality, commutativity and homogeneity conditions respectively. The $\Lambda_{N}^{M}$ 's are $\mathrm{SO}(4 n)$ Lorentz matrices in the $\mathbf{4 n}$ vector representation. If $\mathbf{R}^{4 n}$ is endowed by a complex structure, the natural coordinates are the usual complex variables $z^{\alpha}$ and of $z_{\bar{\alpha}}$ of $\mathbf{C}^{2 n}$. They transform in the fundamental $\mathbf{2 n}$ and $\overline{\mathbf{2 n}}$ representations of $\mathrm{U}(2 n)=\mathrm{U}(1) \times \mathrm{SU}(2 n) \subset \mathrm{SO}(4 n)$ and related to the $x^{M}$ 's as

$$
\begin{aligned}
& z^{\alpha}=x^{\alpha}+i x^{\alpha+2 n} ; \\
& z_{\bar{\alpha}}=x^{\alpha}-i x^{\alpha+2 n}, \quad \alpha=1, \ldots, 2 n .
\end{aligned}
$$

Reality, commutativity and homogeneity of $\mathbf{R}^{4 n}$ then become in $\mathbf{C}^{2 n}$

$$
\begin{array}{r}
{\left[z^{\alpha}, z^{\beta}\right]=\left[z_{\bar{\alpha}}, z_{\bar{\beta}}\right]=\left[z^{\alpha}, z_{\bar{\beta}}\right]=0} \\
z^{\alpha}=u_{. \beta}^{\alpha} z^{\beta}, \quad z_{\bar{\alpha}}^{\prime}=z_{\bar{\beta}} u_{. \bar{\alpha}}^{\bar{\beta}}
\end{array}
$$

where $u_{. \bar{\beta}}^{\bar{\alpha}} \in \mathbf{2 n}$ and $u_{. \bar{\alpha}}^{\bar{\beta}} \in \overline{\mathbf{2 n}}$ with the property $z_{\bar{\alpha}}=\left(z^{\alpha}\right)^{+}$. Though this complex frame exhibits manifestly one complex structure, one can go ahead and study the problem of solving the YM self-duality constraint eqs on $\mathbf{C}^{2}$. One recalls for instance the ADHM construction of YM instantons which find actually many applications in D-brane physics. Using this complex frame one can do even more by considering the problem of $\mathrm{YM}$ fields on $\mathbf{C}^{2 n}, n \geq 1$ and study the solving of the generalized self-dual constraint eqs. Such a kind of problem was considered in many occasions before as in YM and gravitational theories on $\mathbf{C}^{p}$ in connection with the Yang-Lee theory and (hyper)Kahler geometry respectively [34, 35, 27, 36]. In the present study, we shall not use the complex frame as given in (2.2,2.3). What we will do instead is to use the $\mathrm{SU}(2)$ symmetry factor of the $\mathrm{SU}(2) \times \mathrm{SP}(n)$ homogeneity group of $n$ dimensional hyperKahler manifolds to introduce a new local frame of $\mathbf{C}^{2 n}$ where the three complex structures are manifestly exhibited. Breaking the $\mathrm{SO}(4 n)$ Lorentz group of $\mathbf{R}^{4 n}$ down to $\mathrm{SU}(2) \times \mathrm{SP}(n)$ by reindexing the $x^{M}$ variables as $x^{i \alpha}$, where now the double index $(i, \alpha)$ transform in the $(\mathbf{2}, \mathbf{2 n})$ representation of $\mathrm{SU}(2) \times \mathrm{SP}(n)$, eqs (2.1) read then as

$$
\begin{aligned}
\bar{x}^{i \alpha} & =\Omega_{\alpha \beta} \varepsilon_{i j} x^{j \beta}, \\
{\left[x^{i \alpha}, x^{j \beta}\right] } & =0 \\
x^{i \alpha} \rightarrow x^{i \alpha^{\prime}} & =u_{j}^{i} C_{\beta}^{\alpha} x^{j \beta},
\end{aligned}
$$

where $u_{j}^{i}$ and $C_{\beta}^{\alpha}$ are respectively $2 \times 2$ and $2 n \times 2 n$ matrices in the fundamental representations of $\mathrm{SU}(2)$ and $\mathrm{SP}(n)$, while $\Omega$ and $\varepsilon$ satisfy the following properties $\Omega^{\alpha \gamma} \Omega_{\gamma \delta}=\delta_{\delta}^{\gamma}, \quad \Omega^{\alpha \beta}=-\Omega^{\beta \alpha}, \quad \Omega^{\alpha, \alpha+n}=1 ; 1 \leq \alpha \leq n$ and $\varepsilon^{i k} \varepsilon_{k j}=\delta_{j}^{i}, \quad \varepsilon^{i j}=$ $-\varepsilon^{j i}, \varepsilon^{12}=\varepsilon_{21}=1$. 
To write down the generalized self-dual YM constraint eqs using the local coordinates system $\left\{x^{i \alpha}\right\}$, it is enough to consider the gauge covariant derivatives $\mathcal{D}_{i \alpha}$ and the gauge curvatures $F_{i \alpha j \beta}$. Like for YM theory on $\mathbf{R}^{4 n}$ with gauge group $\mathbf{G}$, we have

$$
\begin{aligned}
\mathcal{D}_{i \alpha} & =\frac{\partial}{\partial x^{i \alpha}}+A_{i \alpha} \\
& =\partial_{i \alpha}+A_{i \alpha}
\end{aligned}
$$

where $A_{i \alpha}=A_{i \alpha}\left(x^{j \beta}\right)$ are the gauge potential component fields valued in the Lie algebra $\mathbf{g}$ of the gauge group $\mathbf{G}$. The field strengths $F_{i \alpha j \beta}$ are given by the commutators of the $\mathcal{D}_{i \alpha}$ 's

$$
\left[\mathcal{D}_{i \alpha}, \mathcal{D}_{j \beta}\right]=i F_{i \alpha j \beta} .
$$

Taking the tensor product of the fundamental representation of $S U(2) \times S P(n)$, one can put the above eqs into a convenient form by decomposing $F_{i \alpha j \beta}$ curvatures as shown here below

$$
\left[\mathcal{D}_{i \alpha}, \mathcal{D}_{j \beta}\right]=i \varepsilon_{i j} F_{(\alpha \beta)}+i F_{(i j)[\alpha \beta]},
$$

where ( ) and [] denote complete symmetrisation and antisymmetrisation respectively. Having given the curvatures $F_{i \alpha j \beta}$, we turn now to introduce the generalized selfduality constraint eqs of YM fields on $4 n$ dimensional space with $\mathrm{SU}(2) \times \mathrm{SP}(n)$ homogeneity symmetry. They are defined as

$$
2 i F_{(i j)[\alpha \beta]}=\left(\left[\mathcal{D}_{i \alpha}, \mathcal{D}_{j \beta}\right]+\left[\mathcal{D}_{j \alpha}, \mathcal{D}_{i \beta}\right]\right)=0,
$$

or equivalently

$$
\left[\mathcal{D}_{i \alpha}, \mathcal{D}_{j \beta}\right]=i \varepsilon_{i j} F_{(\alpha \beta)} .
$$

Note that for $n=1$, the homogeneity group of $\mathbf{R}^{4}$ is $\mathrm{SU}(2) \times \mathrm{SP}(1) \sim \mathrm{SO}(4)$. In this case, $F_{(i j)[\alpha \beta]}=\Omega_{\alpha \beta} F_{(i j)}$ and so eq (2.7) becomes

$$
\left[\mathcal{D}_{i \alpha}, \mathcal{D}_{j \beta}\right]=i \varepsilon_{(i j)} F_{(\alpha \beta)}+i \Omega_{\alpha \beta} F_{(i j)} .
$$

In this special situation one may impose not only the self-dual constraint eqs, $F_{(i j)}=0$, but also anti self-dual ones associated with the condition $F_{(\alpha \beta)}=0$.

What we want to do now is to show that in some special situations one may solve explicitly the generalized self-dual constraints (2.8,2.9) by using harmonic analyticity. The method may be summarized as follows: First, instead of using the local coordinates systems $\left\{x^{i \alpha}\right\}$ parameterizing the $4 n$ dimensional homogeneous space $\mathcal{P} / S U(2) \times S P(n)$, where $\mathcal{P}$ stands for the Poincaré group with $\mathrm{SU}(2) \times \mathrm{SP}(n)$ as its Lorentz subgroup, we rather use the harmonic space $\mathcal{P} / \mathrm{SP}(n)$ parametrized by $\left\{x^{ \pm \alpha}, u_{i}^{ \pm}\right\}$. 
On this new space we have the following:

(i) the $u_{i}^{ \pm}$variables parameterize the unit $\mathbf{S}^{3}$ sphere. In terms of these variables, the $\mathrm{SU}(2)$ Lorentz sub-symmetry of the $4 n$ space is generated by the differential operators

$$
\begin{aligned}
D^{++} & =u^{+} \partial / \partial u^{-} \\
D^{--} & =u^{-} \partial / \partial u^{+} \\
D^{0} & =u^{+} \partial / \partial u^{+}-u^{-} \partial / \partial u^{-},
\end{aligned}
$$

which obey the familiar $\mathrm{su}(2)$ algebra

$$
\begin{aligned}
{\left[D^{++}, D^{--}\right] } & =D^{0} \\
{\left[D^{0}, D^{++}\right] } & =2 D^{++} \\
{\left[D^{0}, D^{--}\right] } & =-2 D^{0}
\end{aligned}
$$

(ii) the $x^{+^{\alpha}}$ and $x^{-\alpha}$ bosonic coordinates still carry an $\mathrm{su}(2)$ isospin $\frac{1}{2}$ representation since

$$
\begin{aligned}
{\left[D^{++}, x^{+^{\alpha}}\right] } & =0 \\
{\left[D^{++}, x^{-\alpha}\right] } & =x^{+^{\alpha}} \\
{\left[D^{0}, x^{ \pm \alpha}\right] } & = \pm x^{ \pm \alpha}
\end{aligned}
$$

Eqs (2.13) mean that $x^{+^{\alpha}}=u_{i}^{+} x^{i \alpha}$ and $x^{-\alpha}=u_{i}^{-} x^{i \alpha}$. Note that, as far as the $x^{ \pm}$'s are concerned, the usual reality condition $\widetilde{x^{i \alpha}}=\varepsilon_{i j} \Omega_{\alpha \beta} x^{j \alpha}$ is replaced in HS by

$$
\begin{aligned}
& \widetilde{x^{+^{\alpha}}}=-\Omega_{\alpha \beta} x^{+^{\beta}} \\
& \widetilde{x^{-\alpha}}=\Omega_{\alpha \beta} x^{-\beta} .
\end{aligned}
$$

This conjugaison has been introduced first in [21] and turns out to be the natural conjugation in HS.

(iii) The gauge covariant derivatives in HS language are defined as

$$
\begin{gathered}
\mathcal{D}_{\alpha}^{+}=\partial / \partial x^{-\alpha}+i A_{\alpha}^{+}=\partial_{\alpha}^{+}+i A_{\alpha}^{+} \\
\mathcal{D}_{\alpha}^{-}=\partial / \partial x^{+\alpha}+i A_{\alpha}^{-}=\partial_{\alpha}^{-}+i A_{\alpha}^{-}
\end{gathered}
$$

They obey the following constraint eqs

$$
\begin{aligned}
{\left[D^{++}, \mathcal{D}_{\alpha}^{+}\right] } & =0 \\
{\left[D^{--}, \mathcal{D}_{\alpha}^{+}\right] } & =\mathcal{D}_{\alpha}^{-} \\
{\left[D^{0}, \mathcal{D}_{\alpha}^{ \pm}\right] } & = \pm \mathcal{D}^{ \pm}
\end{aligned}
$$


which ensure that $\mathcal{D}_{\alpha}^{ \pm}$are $\mathrm{SU}(2)$ doublets; i.e. $\mathcal{D}_{\alpha}^{ \pm}=u_{i}^{ \pm} \mathcal{D}_{\alpha}^{i}$.

(iv) In HS, the gauge curvatures read as

$$
\begin{aligned}
& {\left[\mathcal{D}_{\alpha}^{+}, \mathcal{D}_{\beta}^{+}\right]=i F_{[\alpha \beta]}^{++}} \\
& {\left[\mathcal{D}_{\alpha}^{+}, \mathcal{D}_{\beta}^{-}\right]=i F_{(\alpha \beta)}+i F_{[\alpha \beta]}^{+-}}
\end{aligned}
$$

They verify the constraint eqs

$$
\begin{aligned}
{\left[D^{++}, F_{[\alpha \beta]}^{+-}\right] } & =\left[\mathcal{D}^{++}, F_{[\alpha \beta]}^{++}\right]=0 \\
{\left[D^{++}, F_{(\alpha \beta)}\right] } & =0 .
\end{aligned}
$$

Eqs (2.17) mean amongst others that the symmetric part $F_{(\alpha \beta)}$ of the curvature is a $\mathrm{SU}(2)$ singlet as it does not depend on the $u^{ \pm}$'s while the antisymmetric part is a $\mathrm{SU}(2)$ triplet, i.e.

$$
F_{[\alpha \beta]}^{++}=u_{(i}^{+} u_{j)}^{+} F_{[\alpha \beta]}^{(i j)} .
$$

The second step consists of writing the generalized self-duality conditions (2.8,2.9) in the HS as

$$
\left[\mathcal{D}_{\alpha}^{+}, \mathcal{D}_{\beta}^{+}\right]=0 \text {. }
$$

These constraints, which take a simple form in HS, can be treated as integrability conditions for the existence of an analytic harmonic subspace (AHSS) of the HS space. On this subspace, the solving of $F_{[\alpha \beta]}^{++}=0$ may be easily worked out. The key idea of the AHSS resolution method is to say that under a gauge transformation円, one may set $A_{\alpha}^{+}=0$ so that the gauge covariant derivatives $D_{\alpha}^{+}$reduces to a flat space derivative $\partial^{+}=\partial / \partial x^{-\alpha}$. The price one should pay for this operation is that the $\mathrm{su}(2)$ harmonic derivatives $D^{++}, D^{--}$and $D^{0}$ eqs (2.11) acquire now connections as shown here below

$$
\begin{aligned}
D^{++} \rightarrow \mathcal{D}^{++} & =D^{++}+i V^{++} \\
D^{--} \rightarrow \mathcal{D}^{--} & =D^{--}+i V^{--} \\
D^{0} \rightarrow \mathcal{D}^{0} & =D^{0}
\end{aligned}
$$

with the requirement

$$
\begin{aligned}
{\left[\mathcal{D}^{++}, \mathcal{D}^{--}\right] } & =D^{0} \\
{\left[D^{0}, \mathcal{D}^{ \pm \pm}\right] } & = \pm 2 \mathcal{D}^{ \pm \pm}
\end{aligned}
$$

or equivalently

$$
\begin{array}{rlrl}
{\left[D^{0}, V^{++}\right]} & & =2 V^{++}, \\
{\left[D^{0}, V^{--}\right]} & & =-2 V^{--} . \\
D^{++} V^{--}-D^{--} V^{++}+i\left[V^{++}, V^{--}\right] & =0,
\end{array}
$$

\footnotetext{
${ }^{1}$ This transformation is given in [27]
} 
Before going ahead, let us make some remarks regarding this tricky approach. $V^{++}$and $V^{--}$are harmonic gauge connections; they are not independent as they are related through eqs (2.23). Up on solving them, one may usually express $V^{--}$as a function of $V^{++}$. We will show later on how this is done in practice. For the moment let us note that $V^{++}$turns out to be the basic object in solving the generalized selfduality constraint eqs. This property may be easily seen by rewriting the full set of the generalized self-duality constraints in HS. We have:

$$
\begin{aligned}
{\left[D_{\alpha}^{+}, D_{\beta}^{+}\right] } & =0 \\
{\left[D_{\alpha}^{+}, D_{\beta}^{-}\right] } & =i F_{(\alpha \beta)} \\
{\left[D^{0}, D^{ \pm}\right] } & =D_{\alpha}^{ \pm}
\end{aligned}
$$

and

$$
\begin{aligned}
{\left[\mathcal{D}^{++}, D_{\alpha}^{+}\right] } & =0 \\
{\left[\mathcal{D}^{--}, D_{\alpha}^{+}\right] } & =D_{\alpha}^{-} \\
{\left[\mathcal{D}^{++}, \mathcal{D}^{--}\right] } & =D^{0} \\
{\left[\mathcal{D}^{0}, \mathcal{D}^{ \pm \pm}\right] } & = \pm 2 \mathcal{D}^{ \pm \pm}
\end{aligned}
$$

To derive the solutions of these constraints eqs, let us rewrite them in a more convenient way. Using harmonic analyticity which allows to take $\mathcal{D}_{\alpha}^{+}$as $\partial / \partial x^{-\alpha}$; i.e. $A_{\alpha}^{+}=0$, and replacing $\mathcal{D}^{++}, \mathcal{D}^{--}$and $\mathcal{D}^{0}$ by their expressions $(2.21)$, the generalized self-duality constraint eqs may be represented as

$$
\begin{aligned}
& {\left[D^{0}, V^{++}\right] \quad=2 V^{++} \quad(a)} \\
& {\left[\partial_{\alpha}^{+}, V^{++}\right] \quad=0 \quad(b)} \\
& D^{++} V^{--}-D^{--} V^{++}+i\left[V^{++}, V^{--}\right]=0 \quad \text { (c) } \\
& {\left[\partial_{\alpha}^{+}, V^{--}\right] \quad=-A_{\alpha}^{-} \quad(d)} \\
& {\left[\partial_{\alpha}^{+}, A_{\beta}^{-}\right] \quad=F_{(\alpha \beta)} . \quad(e)}
\end{aligned}
$$

These relations define the form of the self-duality YM constraint eqs we are looking for.

\subsection{Solving The Constraints}

To solve the previous eqs, we shall proceed step by step by working out the solution of each relation of the system (2.26). These details will be useful in the study of the instantons in NHS. The first relation (2.26.a) tells us that $V^{++}$is a 
harmonic function on the $\mathbf{S}^{2} \simeq S U(2) / U(1)$ sphere and so it may be expanded in a harmonic series in term of the $u^{ \pm}$'s as:

$$
\begin{aligned}
V^{++}\left(x^{+}, x^{-}, u^{ \pm}\right)= & V^{++}\left(x^{+}, x^{-}\right)+u_{(i}^{+} u_{j)}^{-} V^{++}(i j) \\
& \left.+x_{\left(i_{1}\right.}^{+} u_{i_{2}}^{+} u_{j_{1}}^{-} u_{\left.j_{2}\right)}^{-} V^{++}\right)
\end{aligned}
$$

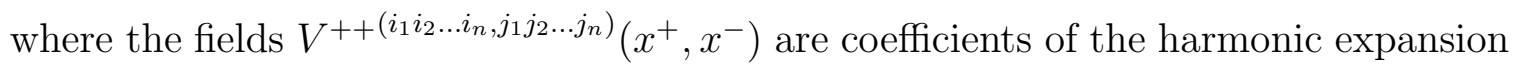
on $\mathbf{S}^{2}$. It turns out that except the leading term $V^{++}\left(x^{+}, x^{-}\right)$where no explicit dependence on the $u^{ \pm}$'s appear, all the extra terms of (2.27) may be ignored due to the existence of $U(1)$ symmetry see (4.24). Forgetting about these terms, and putting (2.27) in (2.26.b), one sees that the potential $V^{++}$should be an analytic function on $\mathbf{S}^{2}$ depending on the $x^{+}$variable only. This means that the general solution of (2.26) for a gauge group $\mathbf{G}$ of generators $\left\{T^{a}\right\}$ should read as

$$
\begin{aligned}
\left(V^{++}\right)^{a} & =\operatorname{tr}\left(T^{a} V^{++}\right) \\
& =\mathcal{M}_{\alpha \beta}^{a} x^{+^{\alpha}} x^{+\beta},
\end{aligned}
$$

where $\mathcal{M}_{\alpha \beta}^{a}$ are constant coefficients scaling as the inverse of the square length dimension. Note that $\mathcal{M}_{\alpha \beta}^{a}$ carry two $\operatorname{SP}(n)$ indices $\alpha, \beta$. Commutativity of $x^{+^{\alpha}}$ and $x^{+\beta}$ requires $\mathcal{M}_{\alpha \beta}^{a}=\mathcal{M}_{\beta \alpha}^{a}$ and so transform under the adjoint representation of the $\mathrm{SP}(n)$ Lorentz subgroup. Note also that in the case where the gauge group $\mathbf{G}$ is itself a $\operatorname{SP}(n)$ symmetry, one may take as a solution for the $\operatorname{SP}(n)$ potentials $V^{++}{ }_{\alpha \beta}$ the following remarkable one

$$
\left(V^{++}\right)_{\alpha \beta}=\frac{i}{\rho^{2}} \Omega_{\alpha \gamma} \Omega_{\beta \delta} x^{+\gamma} x^{+^{\delta}}
$$

where $\rho$ is a dimensionful parameter interpreted in $\mathbf{R}^{4}$ as the size of the $\mathrm{SU}(2)$ instanton. Putting this solution back into the constraint (2.26.c), one gets after some straightforward algebra the following expression for the $\operatorname{SP}(n)$ gauge potentials:

$$
\left(V^{--}\right)_{\alpha \beta}=\frac{i}{\left(x^{2}+\rho^{2}\right)} \Omega_{\alpha \gamma} \Omega_{\beta \delta} x^{-\gamma} x^{-\delta}
$$

where we have used the convention notation $x^{2}=\Omega_{\alpha \beta} x^{+\alpha} x^{-\beta}=x^{+\alpha} x^{-}{ }_{\alpha}$. From (2.30), one gets the gauge potential $A_{\alpha}^{-}$by help of (2.26.d). It reads as:

$$
\left(A_{\nu}^{-}\right)_{\alpha \beta}=\frac{-i}{\left(x^{2}+\rho^{2}\right)}\left[-\Omega_{\nu(\alpha} \Omega_{\beta) \gamma} x^{-\gamma}+\frac{1}{\left(x^{2}+\rho^{2}\right)} \Omega_{\nu \sigma} \Omega_{\alpha \gamma} \Omega_{\beta \delta} x^{+\sigma} x^{-\gamma} x^{-\delta}\right]
$$


The curvatures $\left(F_{\mu \nu}\right)_{\alpha \beta}$ are immediately obtained by solving (2.26.e) using the relation of $A_{\alpha}^{-}$given here above. We find:

$$
\begin{aligned}
\left(F_{\mu \nu}\right)_{\alpha \beta}= & \frac{1}{\left(x^{2}+\rho^{2}\right)}\left[-\Omega_{\mu(\alpha} \Omega_{\beta) \nu}+\frac{1}{\left(x^{2}+\rho^{2}\right)} \Omega_{\mu(\alpha} \Omega_{\beta) \gamma} \Omega_{\nu \sigma} x^{+\sigma} x^{-\gamma}\right] \\
& -\frac{i}{\left(x^{2}+\rho^{2}\right)^{2}} \Omega_{\mu \rho} \Omega_{\nu(\alpha} \Omega_{\beta) \gamma} x^{+\rho} x^{-\gamma} \\
& \frac{2 i}{\left(x^{2}+\rho^{2}\right)^{3}} \Omega_{\mu \rho} \Omega_{\nu \sigma} \Omega_{\alpha \gamma} \Omega_{\beta \delta} x^{+\rho} x^{+\sigma} x^{-\gamma} x^{-\delta}
\end{aligned}
$$

Finally the Lagrangian density of the instanton is:

$$
\operatorname{tr}\left[\left(F_{\mu \nu}\right)^{2}\right]=\frac{\rho^{4}}{\left(x^{2}+\rho^{2}\right)^{4}} .
$$

In the limit $\rho \rightarrow 0$, eq (2.33) has a singularity near the origin and so the density $\operatorname{tr}\left(F_{\mu \nu}\right)^{2}$ behaves as a Dirac delta function.

\section{Yang-Mills Instantons on Noncommutative $\mathbf{R}_{\theta}^{4}$}

We start this section by considering the extension of the harmonic space analyticity idea in order to study the problem of Y-M instantons on noncommutative $\mathbf{R}_{\theta}^{4}$. To do so, we have a priori different choices of non commutative local coordinates. For example one can use directly the usual real coordinates system $\left\{x^{M}\right\}$, where the $x^{M}$ 's transforming in the 4 vector representation of $\mathrm{SO}(4)$ satisfy:

$$
\left[x^{M}, x^{N}\right]=i \theta^{M N}
$$

Then extends the standard analysis of YM instantons by incorporating this noncommutativity feature. An other way is to break the $\mathrm{SO}(4)$ Lorentz group down to $\mathrm{U}(2)=\mathrm{U}(1) \times \mathrm{SU}(2)$ and use the complex coordinates frame $\left\{z^{\mu}=x^{\mu}+i x^{\mu+2} ; i z^{\bar{\mu}}=\right.$ $\left.x^{\mu}-i x^{\mu+2} ; \mu=1,2\right\}$ satisfying the following commutation relations

$$
\begin{aligned}
& {\left[z^{\mu}, z^{\nu}\right]=\theta^{\mu \nu}} \\
& {\left[z^{\bar{\mu}}, z^{\bar{\nu}}\right]=\theta^{\bar{\mu} \bar{\nu}}} \\
& {\left[z^{\mu}, z^{\bar{\nu}}\right]=\theta^{\mu \bar{\nu}}} \\
& {\left[z^{\bar{\mu}}, z^{\nu}\right]=\theta^{\bar{\mu} \nu}}
\end{aligned}
$$

This coordinate system, which is useful whenever one can impose complex analyticity, is nowadays intensively used in the noncommutative ADHM formulation of instantons 
[5, [6, [7]. But we will use neither the first method nor the second. What we will do instead is to develop a non commutative harmonic space analysis by extending the study of subsection 2.2. Thus taking the local coordinates of $\mathbf{R}^{4}$ as $\left\{x^{i \alpha} ; \quad i=\right.$ $1,2 ; \alpha=1,2\}$. The space of noncommutativity reads as

$$
\left[x^{i \alpha}, x^{j \beta}\right]=\Omega^{\alpha \beta} \eta^{(i j)}+\varepsilon^{i j} \theta^{(\alpha \beta)},
$$

where $\eta^{(i j)}$ and $\theta^{(\alpha \beta)}$ transform respectively under the $(1,0)$ and $(0,1)$ representations of the $\mathrm{SU}(2) \times \mathrm{SP}(1)$ Lorentz group. Now introducing the harmonic variables $u_{i}^{ \pm}$, the noncommutative harmonic space is defined as

$$
\begin{aligned}
& {\left[x^{+^{\alpha}}, x^{+\beta}\right]=i \Omega^{\alpha \beta} \eta^{++}} \\
& {\left[x^{-\alpha}, x^{-\beta}\right]=i \Omega^{\alpha \beta} \eta^{--}} \\
& {\left[x^{+^{\alpha}}, x^{-\beta}\right]=i \Omega^{\alpha \beta} \eta-i \theta^{(\alpha \beta)}} \\
& {\left[x^{-\alpha}, x^{+\beta}\right]=i \Omega^{\alpha \beta} \eta+i \theta^{(\alpha \beta)}}
\end{aligned}
$$

where we have used

$$
\begin{aligned}
\eta^{++} & =u_{(i}^{+} u_{j)}^{+} \theta^{(i j)} \\
\eta^{--} & =u_{(i}^{-} u_{j)}^{-} \theta^{(i j)} \\
\eta & =u_{(i}^{+} u_{j)}^{-} \theta^{(i j)} .
\end{aligned}
$$

Observe that according to the values of $\eta^{(i j)}$ and $\theta^{(\alpha \beta)}$ which together carry the six degrees of freedom of the six-dimensional antisymmetric representation of $\mathrm{SO}(4)$, one may distinguish four kinds of harmonic subspaces: (1) the ordinary one considered in section 2 corresponding to $\theta^{(i j)}=0 ; \eta^{(i j)}=0$ and three noncommutative ones given by $(2) \eta^{(i j)} \neq 0 ; \theta^{(\alpha \beta)}=0$, (3) $\eta^{(i j)}=0 ; \theta^{(i j)} \neq 0$ and $(4) \eta^{(i j)} \neq 0 ; \theta^{(\alpha \beta)} \neq 0$. We shall refer to these four kinds of harmonic spaces as $\operatorname{HS}(\eta, \theta)$ which roughly speaking may be viewed as a two deformation parameters of the standard harmonic space. Note moreover that these spaces are intimately related with the problem of finding (anti) self-dual YM instantons on noncommutative geometry. In this regards it is not difficult to see that the noncommutative harmonic subspaces $\operatorname{HS}(\eta, 0)$ and $\operatorname{HS}(0, \theta)$ are respectively associated with self-dual and anti self-dual YM instantons of the noncommutative geometry. The point is to use the Seiberg-Witten realisation according to which in the $\alpha^{\prime}$ zero slope limit the parameters $\eta^{(i j)}$ and $\theta^{(\alpha \beta)}$ are proportional to the inverse of the NS-NS antisymmetric $B_{M N}$ field leaving on an Euclidean D3-Brane world volume. Put differently, if we decompose $B_{i \alpha j \beta}$ as $\varepsilon_{i j} B_{(\alpha \beta)}+\Omega_{\alpha \beta} B_{(i j)}$; we have

$$
\eta^{(i j)} \sim B_{(i j)} / B^{2} ; \quad \theta^{(\alpha \beta)} \sim B_{(\alpha \beta)} / B^{2} .
$$


These eqs show clearly that $\eta^{(i j)}$ is related to the self-dual part of the $B$ field while $\theta^{(\alpha \beta)}$ is given by the anti self-dual one. In what follows we shall consider the example of noncommutative $\operatorname{HS}(\eta, 0)$ and study the problem of finding noncommutative YM instantons by using the idea of harmonic analyticity developed previously. Similar analysis may be done for the anti self-dual constraints by using $\operatorname{NHS}(0, \theta)$.

\subsection{Noncommutative YM instantons in $\operatorname{NHS}(\eta, 0)$}

The commutation relations of the local coordinates $\left\{x^{+^{\alpha}}, x^{-\beta}, u_{i}^{ \pm}\right\}$defining the noncommutative $\operatorname{HS}(\eta, 0)$ are immediately obtained from (3.4) by setting $\theta^{(\alpha \beta)}=0$ which gives

$$
\begin{aligned}
& {\left[x^{+^{\alpha}}, x^{+^{\beta}}\right]=i \Omega^{\alpha \beta} \eta^{++}} \\
& {\left[x^{+^{\alpha}}, x^{-\beta}\right]=i \Omega^{\alpha \beta} \eta} \\
& {\left[x^{-\alpha}, x^{-\beta}\right]=i \Omega^{\alpha \beta} \eta^{--} .}
\end{aligned}
$$

Such commutation relations have however a nice interpretation in D-brane physics in presence of closed string background fields $g_{i \alpha j \beta}$ and $B_{i \alpha j \beta}$. Indeed if we consider a D3-brane with Euclidean $\mathbf{R}^{4}$ world volume in a constant antisymmetric $B$ field and world sheet action

$$
\mathcal{S}=\frac{1}{4 \pi \alpha^{\prime}} \int_{\Sigma}\left(g_{i \alpha j \beta} \delta^{A B}-2 i \pi B_{i \alpha j \beta} \varepsilon^{A B}\right) \partial_{A} x^{i \alpha} \partial_{B} x^{j \beta}
$$

consistency requires that the open string fields $x^{i \alpha}$ should obey the following boundary conditions

$$
\left.\left(g_{i \alpha j \beta} \partial_{n} x^{j \beta}+2 i \alpha^{\prime} B_{i \alpha j \beta} \partial_{t} x^{j \beta}\right)\right|_{\partial \Sigma}=0,
$$

where $\partial_{n}$ and $\partial_{t}$ are the normal and tangential derivatives along the world sheet boundary $\partial \Sigma$ respectively. In practice we will use the classical approximation to open string where $\Sigma$ may be taken as a disc and hence can be conformally mapped to the upper half plane parametrized by $z=\tau+i \sigma$ and $\partial=\frac{1}{2}\left(\frac{\partial}{\partial \tau}-i \frac{\partial}{\partial \sigma}\right), 0 \sigma \geq 0$. In this world sheet coordinates, the boundary conditions (3.9) becomes

$$
\left.\left[g_{i \alpha j \beta}(\partial-\bar{\partial}) x^{j \beta}+2 i \alpha^{\prime} B_{i \alpha j \beta}(\partial+\bar{\partial}) x^{j \beta}\right]\right|_{z=\bar{z}}=0 .
$$

Eq (3.10) describes an interpolation from Neumann to Dirichlet boundary conditions. In the Seiberg Witten limit where $g_{i \alpha j \beta} \sim \alpha^{\prime 2} \varepsilon_{i j} \Omega_{\alpha \beta}$ with $\alpha^{\prime} \sim \epsilon^{\frac{1}{2}}$ goes to zero, Eq (3.10) becomes Dirichlet boundary conditions where at each boundary the open string world sheet is attached to a single point (zero brane) in the D3 brane. In this limit, the action (3.8) reduces to

$$
\mathcal{S}=-\frac{i}{2} \int_{\mathbf{R}} d \tau\left(B_{i j} \Omega^{\alpha \beta} x^{i \alpha} \frac{d x^{j \beta}}{d \tau}+B_{\alpha \beta} \varepsilon_{i j} x^{i \alpha} \frac{d x^{j \beta}}{d \tau}\right) .
$$


Now taking the anti self-dual part $B_{(\alpha \beta)}$ of the antisymmetric NS-NS field to zero, one can calculate the propagators of the boundary fields $x^{i \alpha}(\tau)$. From the eqs of motion of the one dimensional fields $x^{k \gamma}(\tau)$, namely

$$
i \varepsilon^{k i} B_{(i j)} \frac{d x^{j \gamma}}{d \tau}=0,
$$

one can easily check that the propagators of the $x^{i \alpha}(\tau)$ 's read as

$$
\left\langle x^{i \alpha}\left(\tau_{1}\right) x^{j \beta}\left(\tau_{2}\right)\right\rangle=\frac{i}{2} \eta^{(i j)} \Omega^{\alpha \beta} \varepsilon\left(\tau_{1}-\tau_{2}\right),
$$

where $\varepsilon\left(\tau_{1}-\tau_{2}\right)$ is the Heveaside function $\varepsilon(\tau)=1$ for $\tau>0$ and $\varepsilon(\tau)=-1$ for $\tau<0$ and where

$$
\eta^{(i j)} B_{(i j)}=1
$$

In harmonic space $\left\{x^{+^{\alpha}}, x^{-\alpha}, u^{ \pm}\right\}$, the boundary conformal field theory we have discussed is described by the following action

$\mathcal{S}=-\frac{i}{2} \int_{\mathbf{R} \times \mathbf{S}^{2}} d \tau d u \Omega_{\alpha \beta}\left[\left(B^{++} x^{-\alpha} \frac{d x^{-\beta}}{d \tau}-B^{--} x^{+\alpha} \frac{d x^{+\beta}}{d \tau}\right)-B\left(x^{+\alpha} \frac{d x^{-\beta}}{d \tau}+x^{-\alpha} \frac{d x^{+\beta}}{d \tau}\right)\right]$,

where integration with respect to the $u$ 's keeps only $\mathrm{SU}(2)$ singlets and where we have used

$$
\begin{aligned}
B^{++} & =u_{(i}^{+} u_{j)}^{+} B^{(i j)} \\
B^{--} & =u_{(i}^{-} u_{j)}^{-} B^{(i j)} \\
B & =u_{(i}^{+} u_{j)}^{-} B^{(i j)} .
\end{aligned}
$$

The propagators in the harmonic space are

$$
\begin{aligned}
\left\langle x^{+i \alpha}\left(\tau_{1}\right) x^{+j \beta}\left(\tau_{2}\right)\right\rangle & =\frac{i}{2} \Omega^{\alpha \beta} \eta^{++} \varepsilon\left(\tau_{12}\right), \\
\left\langle x^{-i \alpha}\left(\tau_{1}\right) x^{-j \beta}\left(\tau_{2}\right)\right\rangle & =\frac{i}{2} \Omega^{\alpha \beta} \eta^{--} \varepsilon\left(\tau_{12}\right), \\
\left\langle x^{+i \alpha}\left(\tau_{1}\right) x^{-j \beta}\left(\tau_{2}\right)\right\rangle & =\frac{i}{2} \Omega^{\alpha \beta} \eta \varepsilon\left(\tau_{12}\right), \\
\left\langle x^{-i \alpha}\left(\tau_{1}\right) x^{+j \beta}\left(\tau_{2}\right)\right\rangle & =\frac{i}{2} \Omega^{\alpha \beta} \eta \varepsilon\left(\tau_{12}\right),
\end{aligned}
$$

Computing the commutators of the conformal field operators $x^{ \pm \alpha}(\tau)$ by using the short distance products (3.17), we find the following relations

$$
\left[x^{+i \alpha}\left(\tau_{1}\right), x^{+j \beta}\left(\tau_{2}\right)\right]=i \Omega^{\alpha \beta} \eta^{++},
$$




$$
\begin{aligned}
& {\left[x^{-i \alpha}\left(\tau_{1}\right), x^{-j \beta}\left(\tau_{2}\right)\right]=i \Omega^{\alpha \beta} \eta^{--},} \\
& {\left[x^{+i \alpha}\left(\tau_{1}\right), x^{-j \beta}\left(\tau_{2}\right)\right]=i \Omega^{\alpha \beta} \eta} \\
& {\left[x^{-i \alpha}\left(\tau_{1}\right), x^{+j \beta}\left(\tau_{2}\right)\right]=i \Omega^{\alpha \beta} \eta}
\end{aligned}
$$

which are similar to the commutation relations of the noncommutative harmonic space $\operatorname{NHS}(\eta, 0)$ given by (3.4). In the remainder of this section we want to discuss some features of the algebra of functions on $\operatorname{NHS}(\eta, 0)$. In conformal field theory on harmonic space one distinguishes different ground state vertex operators;

$$
\exp \left( \pm i p^{+} x^{-}\right) ; \quad \exp \left( \pm i p^{-} x^{+}\right)
$$

They satisfy the short distance products

$$
\begin{aligned}
& : e^{+i p^{-} x^{+}}(1):: e^{\left.+i q^{-} x^{+}\right)}(2):=e^{-\frac{1}{2} \eta^{++} p^{-} q^{-}}: e^{i\left(p^{-}+q^{-}\right) x^{+}}(2): \\
& : e^{-i p^{+} x^{-}}(1):: e^{\left.-i q^{+} x^{-}\right)}(2):=e^{-\frac{1}{2} \eta^{--} p^{+} q^{+}}: e^{i\left(p^{+}+q^{+}\right) x^{-}}(2): \\
& : e^{+i p^{-} x^{+}}(1):: e^{\left.-i q^{+} x^{-}\right)}(2):=e^{\frac{1}{2} \eta p^{-} q^{+}}: e^{+i\left(p^{-} x^{+}-q^{+} x^{-}\right)}(2): \\
& : e^{-i p^{+} x^{-}}(1):: e^{\left.-i q^{-} x^{+}\right)}(2):=e^{\frac{1}{2} \eta p^{+} q^{-}}: e^{-i\left(p^{+} x^{-}-q^{-} x^{+}\right)}(2): .
\end{aligned}
$$

In noncommutative harmonic space language, the above short distance products coincide with the usual star product on noncommutative geometry. Thus we have for instance the identification

$$
\lim _{\tau \rightarrow 0} e^{ \pm i p^{-} x^{+}}(\tau) \cdot e^{ \pm i q^{-} x^{+}}(0) \sim e^{ \pm i p^{-} x^{+}} * e^{ \pm i q^{-} x^{+}}
$$

More generally given two harmonic space functions $f^{\left(q_{1}\right)}\left(x^{+}, x^{-}, u\right)$ and $g^{\left(q_{2}\right)}\left(y^{+}, y^{-}, u\right)$, the star product of these functions is defined as

$$
f^{\left(q_{1}\right)}(x) * g^{\left(q_{2}\right)}(y)=\exp \frac{i}{2}\left(\Omega^{\alpha \beta} M_{\alpha \beta}\right) f^{\left(q_{1}\right)}(x) g^{\left(q_{2}\right)}(y)
$$

where

$$
M_{\alpha \beta}=\left(\eta^{++} \frac{\partial}{\partial x^{+\alpha}} \frac{\partial}{\partial y^{+\beta}}+\eta^{--} \frac{\partial}{\partial x^{-\alpha}} \frac{\partial}{\partial y^{-\beta}}\right)-\eta\left(\frac{\partial}{\partial x^{+\alpha}} \frac{\partial}{\partial y^{-\beta}}+\frac{\partial}{\partial x^{-\alpha}} \frac{\partial}{\partial y^{+\beta}}\right) .
$$

At first order in $\eta^{++}, \eta^{--}$and $\eta$, (3.22) reduces to

$$
\begin{aligned}
f^{\left(q_{1}\right)} * g^{\left(q_{2}\right)}= & f^{\left(q_{1}\right)} g^{\left(q_{2}\right)}+\frac{i}{2} \Omega^{\alpha \beta}\left[\left(\eta^{++} \partial_{\alpha}^{-} f^{\left(q_{1}\right)} \partial_{\beta}^{-} g^{\left(q_{2}\right)}+\eta^{--} \partial_{\alpha}^{+} f^{\left(q_{1}\right)} \partial_{\beta}^{+} g^{\left(q_{2}\right)}\right)\right. \\
& \left.-\eta\left(\partial_{\alpha}^{-} f^{\left(q_{1}\right)} \partial_{\beta}^{+} g^{\left(q_{2}\right)}+\partial_{\alpha}^{+} f^{\left(q_{1}\right)} \partial_{\beta}^{-} g^{\left(q_{2}\right)}\right)\right]+\mathcal{O}(2)
\end{aligned}
$$




\subsection{Self-Dual Yang-Mills Constraints in $\operatorname{NHS}(\eta, 0)$}

Noncommutative YM theory in Euclidean four dimensional space is formulated in a similar way as YM theory in ordinary $\mathbf{R}^{4}$ except that the gauge group matrix multiplication is now replaced by the star product (3.22). For instance the transformations of the gauge field $\widehat{A}_{M}$ and the field strength $\widehat{F}_{M N}$ of noncommutative YM theory are

$$
\begin{aligned}
\delta_{\widehat{\tau}} \widehat{A}_{\mu} & =\partial_{\mu} \widehat{\tau}-i\left(\widehat{\tau} * \widehat{A}_{\mu}-\widehat{A}_{\mu} * \widehat{\tau}\right) \\
\widehat{F}_{M N} & =\partial_{M} \widehat{A}_{N}-\partial_{N} \widehat{A}_{M}-i\left(\widehat{A}_{M} * \widehat{A}_{N}-\widehat{A}_{N} * \widehat{A}_{M}\right) \\
\delta_{\widehat{\tau}} \widehat{F}_{M N} & =i\left(\widehat{\tau} * \widehat{F}_{M N}-\widehat{F}_{M N} * \widehat{\tau}\right) .
\end{aligned}
$$

Remark that the gauge parameters $\widehat{\tau}$, the fields $\widehat{A}_{M}$ and $\widehat{F}_{M N}$ carry a hat in order to be distinguished from their ordinary geometry analogue. This convention notation will also be used in the remainder of this study.

YM gauge theory in noncommutative harmonic space may be constructed in a similar manner as in the standard formulation. This is achieved in practice by extending the usual $\mathrm{HS}(0,0)$ classical fields to functionals on $\operatorname{NHS}(\eta, 0)$ and replacing the ordinary product by the star one given by (3.22). The novelty brought by the harmonic variables is completely controlled by the SU(2) symmetry. Since the $u_{i}^{ \pm}$ variables still obey the same relations as in ordinary $\operatorname{HS}(0,0)$, the covariant harmonic derivatives in $\operatorname{NHS}(\eta, 0)$ defined as

$$
\begin{aligned}
\mathcal{D}^{++} & =D^{++}+i \widehat{V}^{++} \\
\mathcal{D}^{--} & =D^{--}+i \widehat{V}^{--} \\
\mathcal{D}^{0} & =D^{0}
\end{aligned}
$$

where $\widehat{V}^{++}$and $\widehat{V}^{--}$are harmonic gauge connections on $\operatorname{HS}(\eta, 0)$, still obey the $\mathrm{SU}(2)$ algebra (2.22) which requires in turns:

$$
\begin{array}{rlrl}
{\left[D^{0}, \widehat{V}^{++}\right]} & & =2 \widehat{V}^{++} ; \\
{\left[D^{0}, \widehat{V}^{--}\right]} & & =-2 \widehat{V}^{--} \\
D^{++} \widehat{V}^{--}-D^{--} \widehat{V}^{++}+i\left(\widehat{V}^{++} * \widehat{V}^{--}-\widehat{V}^{--} * \widehat{V}^{++}\right) & =0 .
\end{array}
$$

The $\mathrm{SU}(2)$ symmetry (2.22) and (3.26, 3.27) shows also that the self-dual YM constraint eqs in noncommutative $\mathrm{NHS}(\eta, 0)$ may be obtained from $(2.26)$ by replacing the $\operatorname{HS}(0,0)$ objects by their extensions on $\operatorname{NHS}(\eta, 0)$. The noncommutative self-dual YM constraints read then:

$$
\left[D^{0}, \widehat{V}^{++}\right] \quad=2 \widehat{V}^{++}
$$




$$
\begin{array}{rll}
{\left[\partial_{\alpha}^{+}, \widehat{V}^{++}\right]} & =0 & (b) \\
D^{++} \widehat{V}^{--}-D^{--} \widehat{V}^{++} & =-i\left(\widehat{V}^{++} * \widehat{V}^{--}-\widehat{V}^{--} * \widehat{V}^{++}\right) \\
{\left[\partial_{\alpha}^{+}, \widehat{V}^{--}\right]} & =-\widehat{A}_{\alpha}^{-} & (d) \\
{\left[\partial_{\alpha}^{+}, \widehat{A}_{\beta}^{-}\right]} & =\widehat{F}_{(\alpha \beta)} & (e)
\end{array}
$$

To solve these constraint eqs, we shall make two analysis by considering first perturbative solutions around the ordinary one. Then we examine the exact solution of these constraints by using noncommutative calculus on $\operatorname{NHS}(\eta, 0)$.

\subsection{Perturbative Analysis}

Here we shall consider special perturbative solutions preserving manifestly the $\mathrm{SU}(2)$ symmetry and corresponding to small values of the deformation parameters $\eta^{++}, \eta^{--}$and $\eta$. Eqs (3.28) suggest that one may expand the noncommutative connections $\widehat{V}^{++}$and $\widehat{V}^{--}$around the ordinary $V^{++}$and $V^{--}$ones of $(2.29,2.30)$ as follows

$$
\begin{aligned}
\widehat{V}^{++} & =V^{++}+\eta W^{++}+\mathcal{O}(2) \\
\widehat{V}^{--} & =V^{--}+\eta U^{--}+\eta^{--} U+\eta^{++} U^{-4}+\mathcal{O}(2)
\end{aligned}
$$

Expanding (3.28.c) to first order in $\eta^{++}, \eta^{--}$and $\eta$ as in (3.22) and using (3.28 3.29), in particular the analyticity of $\widehat{V}^{++}$, we find

$$
\begin{aligned}
\eta & \left(D^{++} U^{--}+2 U\right)+\eta^{--} D^{++} U \\
& +\eta^{++}\left(D^{++} U^{-4}+U^{--}\right)-\eta D^{--} W^{++}-\eta^{--} W^{++} \\
& =-i\left(\eta\left\{\left[V^{++}, U^{++}\right]+\left[W^{++}, V^{--}\right]\right\}\right. \\
& \left.+\eta^{--}\left[V^{++}, U\right]+\eta^{++}\left[V^{++}, U^{-4}\right]\right)+\frac{1}{2} \Omega^{\alpha \beta}\left\{\eta ^ { + + } \left(\partial_{\alpha}^{-} V^{++} \partial_{\beta}^{-} V^{--}\right.\right. \\
& \left.\left.-\partial_{\alpha}^{-} V^{--} \partial_{\beta}^{-} V^{++}\right)-\eta\left(\partial_{\alpha}^{-} V^{++} \partial_{\beta}^{+} V^{--}-\partial_{\alpha}^{+} V^{--} \partial_{\beta}^{-} V^{++}\right)\right\}+\mathcal{O}(2) .
\end{aligned}
$$

These eqs imply in turn

$$
\begin{gathered}
D^{++} U-W^{++}+i\left[V^{++}, U\right]=0 \\
D^{++} U^{--}-D^{--} W^{++}+2 U+i\left[V^{++}, U^{--}\right]+i\left[W^{++}, V^{--}\right]=\frac{1}{2} \Omega^{\alpha \beta}\left\{\partial_{\alpha}^{-} V^{++} \partial_{\beta}^{+} V^{--}\right. \\
\left.-\partial_{\alpha}^{+} V^{--} \partial_{\beta}^{-} V^{++}\right) \\
D^{++} U^{-4}+i\left[V^{++}, U^{-4}\right]+U^{--}=\frac{1}{2} \Omega^{\alpha \beta}\left\{\partial_{\alpha}^{-} V^{++} \partial_{\beta}^{-} V^{--}\right. \\
\left.-\partial_{\alpha}^{-} V^{--} \partial_{\beta}^{-} V^{++}\right)
\end{gathered}
$$


To solve these eqs, we choose the fields $W^{++}, U, U^{--}$and $U^{-4}$ of the form

$$
\begin{aligned}
W_{\beta}^{++\alpha} & =a x^{+\alpha} x_{\beta}^{+} \\
U_{\beta}^{\alpha} & =b_{1} x^{+{ }^{\alpha}} x_{\beta}^{-}+b_{2} x^{-\alpha} x^{+}{ }_{\beta} \\
U^{--\alpha} & =c x^{-\alpha} x^{-}{ }_{\beta}
\end{aligned}
$$

where $a, b_{1}, b_{2}$ and $c$ are parameters to be determined. Lengthy but straightforward calculations lead to:

$$
\begin{aligned}
a & =\frac{1}{\rho^{4}} \\
b_{1} & =-\frac{1}{2\left(x^{2}+\rho^{2}\right)^{2}} \\
b_{2} & =-\frac{2 x^{2}+\rho^{2}}{2 \rho^{2}\left(x^{2}+\rho^{2}\right)^{2}} \\
c & =-\frac{1}{\left(x^{2}+\rho^{2}\right)^{2}} .
\end{aligned}
$$

\section{$4 \quad$ Exact Solution}

Here we give the exact solution of the self-dual YM constraint (3.28) on noncommutative $\operatorname{HS}(\eta, 0)$. As for ordinary $\operatorname{HS}(0,0)$ harmonic space, (3.28. a-b) show that $\widehat{V}^{++}$is a harmonic function on $\operatorname{NHS}(\eta, 0)$ depending on $x^{+^{\alpha}}$ only. This means that according to (3.28.a-b), $\widehat{V}^{++}$may be written as

$$
\left(V^{++}\right)^{\alpha \beta}=x^{+^{\alpha}} * A * x^{+\beta}+C^{++} \Omega^{\alpha \beta}
$$

where $A$ and $C^{++}$are harmonic functions without any dependence on the $x^{ \pm}$'s. The $A$ and $C^{++}$scale as the inverse of length squared. Therefore, they behave as the inverse of $\eta$ since according to (3.4)), one can check that we have the following identities

$$
\begin{aligned}
x^{+}{ }_{\gamma} * x^{+\gamma} & =x^{+} * x^{+}=i \eta^{++} \\
x^{-}{ }_{\gamma} * x^{-\gamma} & =x^{-} * x^{-}=i \eta^{--} \\
x^{+}{ }_{\gamma} * x^{-\gamma} & =x^{+} * x^{-}=i \eta+z^{2} \\
x^{-}{ }_{\gamma} * x^{+\gamma} & =x^{-} * x^{+}=i \eta-z^{2},
\end{aligned}
$$

where we have used $\Omega_{\alpha \beta} \Omega^{\alpha \beta}=2$ and set $z^{2}=\frac{1}{2}\left(x^{+} * x^{-}-x^{-} * x^{+}\right) \geq 0$. Note that (4.1,4.2) satisfy

$$
\begin{aligned}
D^{--}\left(V^{++}\right)^{\alpha \beta}= & \left(x^{+^{\alpha}} * A * x^{-\beta}+x^{-\alpha} * A * x^{+\beta}\right) \\
& +x^{+^{\alpha}} *\left(D^{--} A\right) * x^{+\beta}+\left(D^{--} C^{++}\right) \Omega^{\alpha \beta} .
\end{aligned}
$$


and

$$
\begin{aligned}
D^{++} \eta^{++} & =0 \\
D^{++} \eta & =\eta^{++} \\
D^{++} \eta^{--} & =2 \eta .
\end{aligned}
$$

The next step is to find $\left(V^{--}\right)^{\alpha \beta}$ by solving (3.28.c). Harmonic analysis on $N H S(\eta, 0)$ shows that we should look for a solution of the form

$$
\begin{aligned}
\left(V^{--}\right)^{\alpha \beta}= & x^{-\alpha} * B * x^{-\beta}+x^{+^{\alpha}} * E^{--} * x^{-\beta} \\
& +x^{-\alpha} * K^{--} * x^{+\beta}+x^{+^{\alpha}} * G^{(-4)} * x^{+^{\beta}}+H^{--} \Omega^{\alpha \beta}
\end{aligned}
$$

where $B, E^{--}, K^{--}, G^{(-4)}$ and $H^{--}$are to be determined . Taking the harmonic derivative of (4.5), we get

$$
\begin{aligned}
D^{++}\left(V^{--}\right)^{\alpha \beta}= & x^{+^{\alpha}} *\left[B+D^{++} E^{--}\right] * x^{-\beta}+x^{-\alpha} *\left[B+D^{++} K^{--}\right] * x^{+\beta} \\
& +x^{+^{\alpha}} *\left[E^{--}+K^{--}+D^{++} G^{(-4)}\right] * x^{+\beta} \\
& +x^{-\alpha} *\left[D^{++} B\right] * x^{-\beta}+D^{++} H^{--} \Omega^{\alpha \beta}
\end{aligned}
$$

Moreover using (4.1) and (4.5) we have

$$
\begin{aligned}
i\left(V_{\gamma}^{++\alpha} * V_{\beta}^{--\gamma}-\right. & \left.V_{\gamma}^{--\alpha} * V_{\beta}^{++\gamma}\right) \\
= & x^{+\alpha} *\left[i A *\left(x^{+} x^{-}\right) * B+i A *\left(x^{+} x^{+}\right) * E^{--}\right] * x^{-\beta} \\
& +x^{-\alpha} *\left[-i B *\left(x^{-} x^{+}\right) * A-i K^{--} *\left(x^{+} x^{+}\right) * A\right] * x^{+\beta}(4.7) \\
& +x^{+\alpha} *\left[i A *\left(x^{+} x^{-}\right) * K^{--}+i A *\left(x^{+} x^{+}\right) * G^{(-4)}\right. \\
& \left.-E^{--} *\left(x^{-} x^{+}\right) * A-i G^{(-4)} *\left(x^{+} x^{+}\right) * A\right] * x^{+^{\beta}} .
\end{aligned}
$$

Putting back (4.1.4.4),(4.5) and (4.6) in the constraint (3.28.c) one gets an equation transforming in the $\mathbf{1} \oplus \mathbf{3}$ representation of the $\mathrm{SU}(2)$ symmetry which up on projecting it along the different $\mathrm{U}(1)$ cartan subsymmetry directions, we get the following system of four equations

$$
\begin{array}{ccc}
{\left[D^{++} E^{--}+i A *\left(x^{+} x^{+}\right) * E^{--}+\left[1+i A *\left(x^{+} x^{-}\right)\right] * B-A\right]} & =0(a) \\
\left.\left[D^{++} K^{--}-i K^{--} *\left(x^{+} x^{+}\right) * A+B *\left[1-i A *\left(x^{-} x^{+}\right)\right] * A\right]-A\right] & =0(b) \\
{\left[D^{++} G^{(-4)}+E^{--} *\left(1-i\left(x^{-} x^{+}\right) * A\right)\right.} & \\
\left.+\left[1+i A *\left(x^{+} x^{-}\right)\right] * K^{--}-D^{--} A\right] & =0(c) \\
D^{++} B & =0(d) \\
D^{++} H^{--}-D^{--} C^{++} & =0(e)
\end{array}
$$


To solve these, it is interesting to note the following: (1) the $\eta$ parameter is a $\mathbf{C}$ number independent of the $x^{ \pm}$'s and so commute with the star product. it scales as (length) $)^{-2} \cdot \eta^{--}$and $\eta^{++}$are proportional to the norm of the $\operatorname{sp}(1)$ isospinor $x^{+^{\alpha}}$ and $x^{-\alpha}$ which are no longer zero due to noncommutativity. (2) In the limit $\eta \rightarrow 0$, $x^{+} x^{-} \geq 0$ and $x^{-} x^{+} \leq 0$ as may be check explicitly by help of (2.4) (3) Dimensional arguments show that the unknown functions $A, B, C^{--}, E^{--}, K^{--}$and $G^{(-4)}$ in (4.8) scale as

$$
\begin{aligned}
{[A] } & =[B]=\left[E^{--}\right]=\left[K^{--}\right]=\left[G^{-4}\right]=-[\eta] \\
& =-2\left[x^{ \pm}\right]=(\text {length })^{-2}
\end{aligned}
$$

(4) In the limit $\eta \rightarrow 0$, eqs (4.8) should coincide with the ordinary case and so

$$
\begin{aligned}
\lim _{\eta \rightarrow 0} A & =-i \frac{1}{\rho^{2}} \\
\lim _{\eta \rightarrow 0} B & =-i \frac{1}{\rho^{2}+z^{2}} \\
\lim _{\eta \rightarrow 0} E^{--} & =\lim _{\eta \rightarrow 0} K^{--}=\lim _{\eta \rightarrow 0} G^{(-4)}=0 .
\end{aligned}
$$

These limits show that $E^{--}, K^{--}$and $G^{-4}$ should be proportional to $\eta$. Eq (4.8.d) shows that $B$ does not depend on the harmonics. This means that if it carries a dependence on $\eta$, then this should be in terms of the invariant $\eta^{++} \eta^{--}-\eta^{2}=$ $\eta^{(i j)} \eta_{i j}=\vec{\eta}^{2}$. However the dimensional arguments show that the natural solution is just the ordinary one which reads as

$$
B=-i \frac{2}{\left(2 \rho^{2}+x^{+} x^{-}-x^{-} x^{+}\right)}=-i \frac{1}{\rho^{2}+z^{2}},
$$

where we have used (4.2).

Now we turn to solve (4.8. a-b) which we rewrite using (4.2) as

$$
\begin{aligned}
{\left[D^{++} E^{--}-\eta^{++} A E^{--}\right]+\left[\left(1+i A x^{+} x^{-}\right) B-A\right] } & =0 \\
{\left[D^{++} K^{--}-\eta^{++} A K^{--}\right]+\left[\left(1-i A x^{-} x^{+}\right) B-A\right] } & =0
\end{aligned}
$$

Taking $A$ to be

$$
A=-i \frac{1}{\rho^{2}-i \eta}
$$

and using (4.11), the last terms of eq (4.2.a) vanish. It follows that (4.12) become

$$
\begin{array}{cc}
\left(D^{++}+i \frac{\eta^{++}}{\rho^{2}-i \eta}\right) E^{--} & =0 \\
\left(D^{++}-i \frac{\eta^{++}}{\rho^{2}-i \eta}\right) K^{--}+2 \eta A B & =0 .
\end{array}
$$


Integrating these eqs, we find that the most general solution is given by

$$
\begin{aligned}
E^{--} & =\operatorname{cnst} \frac{\rho^{2}-i \eta}{\left(\vec{\eta}^{2}+\eta^{2}\right)} \\
K^{--} & =-\eta^{--} A B \\
& =\frac{\eta^{--}}{\left(\rho^{2}-i \eta\right)\left(2 \rho^{2}+x^{+} x^{-}-x^{-} x^{+}\right)}
\end{aligned}
$$

However the constant appearing in the solution of (4.15) should vanish due to the constraints (4.10). Therefore

$$
E^{--}=0
$$

Now we turn to solve 4.8.c). Using the previous solutions (4.11), (4.13), (4.15) and (4.16), eq (4.8.c) can be brought to the following form

$$
D^{++} G^{(-4)}+\frac{A}{B} K^{--}=D^{--} A .
$$

At first sight, this eq seems to be difficult to handle; but if we consider the following remarkable features

$$
\begin{aligned}
K^{--} & =-A B \eta^{--} \\
D^{--} A & =-\eta^{--} A^{2}
\end{aligned}
$$

it reduces to

$$
D^{++} G^{(-4)}=0
$$

and so

$$
G^{(-4)}=0
$$

Finally, concerning (4.8.e), the $H^{--}$and $C^{++}$are solved as

$$
\begin{aligned}
& C^{++}=D^{++} \lambda \\
& H^{--}=D^{--} \lambda,
\end{aligned}
$$

where $\lambda$ is an arbitrary function on the $\mathbf{S}^{2}$.

\section{Summary}

The noncommutative $\mathrm{YM} \mathrm{SU}(2)$ instanton formulated on $\operatorname{NHS}(\eta, 0)$ is described by the harmonic connections $V_{\beta}^{++\alpha}$ and $V_{\beta}^{--\alpha}$ given

$$
\begin{aligned}
V_{\beta}^{++\alpha}= & x^{+\alpha} * A * x^{+}{ }_{\beta}+D^{++} \lambda \delta_{\beta}^{\alpha} \\
V_{\beta}^{--\alpha}= & x^{-\alpha} * B * x^{-}{ }_{\beta}-\eta^{--} x^{-\alpha} * A * B * x^{+}{ }_{\beta} \\
& +D^{--} \lambda \delta_{\beta}^{\alpha}
\end{aligned}
$$


with

$$
\begin{aligned}
A & =-i \frac{1}{\rho^{2}-i \eta} \\
B & =-i \frac{1}{\rho^{2}-i \eta+x^{+} x^{-}} .
\end{aligned}
$$

At this level certain interesting comments may be done: (1) The solutions of the noncommutative YM self-dual constraint eqs we have obtained are exact solutions. They extend the perturbative one given in the end of section 3 .

(2) our solution admits a $\mathrm{U}(1)$ symmetry;

$$
\begin{aligned}
& V^{++} \rightarrow V^{++}+D^{++} \lambda \\
& V^{--} \rightarrow V^{--}+D^{--} \lambda
\end{aligned}
$$

for any $\lambda$. (3) As long as $\eta \neq 0$, our solution is non singular. This result agrees with the absence of small instanton in noncommutative geometry. (4) In the limit $\eta \rightarrow 0$, we recover the ordinary solution. Using the associativity of the star product, (3.22) and the algebra of the differential operators

$$
\begin{aligned}
\nabla_{\mu}^{-} & =\eta^{--} \partial_{\mu}^{+}-\eta \partial_{\mu}^{-} \\
\nabla_{\mu}^{+} & =\eta^{++} \partial_{\mu}^{-}-\eta \partial_{\mu}^{+}
\end{aligned}
$$

one discovers the perturbative solution obtained in section 3 (3.29 3.33). (5) In the ordinary geometry, the parameter $\rho^{2}$ is interpreted as the size of the YM instantons. It is also a real Kahler parameter of the resolution of ADE singularities by blowing up two-spheres. In the noncommutative YM theory in $\operatorname{NHS}(\eta, 0), \rho^{2}$ is shifted by $\eta$ and becomes a quaternionic parameter $\varrho$ as shown here below

$$
\begin{aligned}
\varrho & =\rho^{2}-i \eta \\
& =\rho^{2}-i u_{(k}^{+} u_{l)}^{-} \eta^{(k l)} .
\end{aligned}
$$

(6) Finally observe that it is possible to work out an other solution of the constraint (3.28) by taking

$$
A=-i \frac{1}{\rho^{2}+i \eta} .
$$

This choice affects (4.12) which becomes

$$
\begin{aligned}
\left(D^{++}+i \frac{\eta^{++}}{\rho^{2}+i \eta}\right) E^{--}-2 \eta A B & =0 \\
\left(D^{++}+i \frac{\eta^{++}}{\rho^{2}+i \eta}\right) K^{--} & =0 .
\end{aligned}
$$

The second class of solutions of (3.28) are given by (4.22) and (4.23) up to performing $\eta \rightarrow-\eta$ and $K^{--} \leftrightarrow E^{--}$. 


\section{Conclusion}

In this paper we have studied Yang-Mills Instantons on noncommutative harmonic space $\operatorname{NHS}(\eta, 0)$. This approach has the advantage of allowing to explicit exact solutions of the noncommutative self dual Yang Mills constraint eqs. It also has the merit of going beyond the perturbative solution described in [1].

We have first developed harmonic space noncommutative geometry and have shown that $\operatorname{NHS}(\eta, \theta)$ has two remarkable NHS subspaces in addition to the usual ordinary one. This property may be seen by remarking that $\mathrm{NHS}(\eta, \theta)$ has two deformation parameters $\eta$ and $\theta$ transforming as $(1,0)$ and $(0,1)$ representations of $\mathrm{SU}(2) \times \mathrm{SP}(1) \sim \mathrm{SO}(4)$ Lorentz group. According to whether $\eta$ and $\theta$ are zero or not, we obtain four subspaces, three of them are noncommutative. Second we have reformulated the noncommutative self-duality Yang-Mills constraints in $\operatorname{NHS}(\eta, \theta)$ by extending the idea of harmonic analyticity. In this formulation, the basic objects carrying the Yang-Mills self-dual constraints are given by the harmonic connections $\widehat{V}^{++}$and $\widehat{V}^{--}$of a gauged $\mathrm{SU}(2)$ symmetry. The latter is a generalisation of a well known trick allowing to go from the standard analysis to the harmonic one. The noncommutative gauge fields $\widehat{A}_{M}$ and curvatures $\widehat{F}_{\mu \nu}$ are related to $\widehat{V}^{++}$and $\widehat{V}^{--}$ as shown in eqs (3.28). Third we have studied the solutions of (3.28) by considering in a first step perturbative solutions around the ordinary one. In a second step, we have given an explicit derivation of an exact solution of the self-dual Yang-Mills constraints. It should be noted here that besides the power of harmonic space analysis,

this exact solution has been made possible due to the choice of $\theta^{(\alpha \beta)}=0$ and useful properties described in section 4 .

\section{Acknowledgements}

Belhaj would like to thank the organizers of the Spring Workshop on Superstrings and related Matters ( March 2000), The Abdus Salam International Centre for theoretical Physics Trieste, Italy for hospitality ; where a part of this work is done. He is grateful to N. Nekrasov and S. Kachru for valuable discussions.

Sahraoui would like to thank DFG under contract 445 Mar 113/5/0 to have supported his stay at the university of Muenchen, Pro. J. Wess to have invited him and Dr. R. Dick for his kindness and hospitality. Many thanks for Pro S. Theisen for his helpful discussions and suggestions.

This research is supported by the program SARS 99/2000. contract CNCPRSTUniversité Mohammed V, Rabat. 


\section{References}

[1] N. Seiberg and E. Witten, "String Theory and Noncommutative geometry"; JHEP 9909 (1999) 032, hep-th/9908142.

[2] A. Connes, M. R. Douglas and A. Schwarz, "Noncommutative Geometry and Matrix theory : Compactification on torus"; Adv. Theor. Math Phys. 1 (1998) 35. hep-th/9711162.

[3] P.-M. Ho, Y.-Y. Wu and Y.-S. Wu, "Towards a Noncommutative Geometric Approach to Matrix Compactifications"; Phys .Rev.D 58 (1998) 026006, hep-th/9712201.

[4] I. Benkadour, M. Bennai, E. Y. Diaf and E. H. Saidi, "On Matrix model Compactification on non commutative $F_{0}$ geometry"; Class . Quant. Grav 17(2000)

[5] N. Nekrasov and A. Schwarz, "Instanton on Non Commutative $R^{4}$ and $(2,0)$ Superconformal Six dimensional theory"; Commun Math. Phys 198(1998) 689703 hep-th/9802068.

[6] H. W. Braden, N. Nekrasov, "Space -Time Foam Non-Commutative Instanton"; hep-th/9912019.

[7] N. Nekrasov, "lecture Notes at Spring Workshop ICTP Trieste"; March 2000

[8] N. Seiberg and E. Witten, "D1-D5 and singular CFT"; JHEP 9904 (1999) 017,hep-th/9903224.

[9] O. Aharony, M. Berkooz"IR Dynamics of $\mathrm{d}=2, \mathrm{~N}=(4,4)$ Gauge Theories and DLCQ of Little String theories"; JHEP 9910 (1999) 0030, hep-th/9909101.

[10] A. Belhaj . El Fallah and E.H.Saidi; Class. Quant. Grav 16 (1999)3297 A. Belhaj . El Fallah and E.H.Saidi; Class. Quant. Grav 17 (2000)515

A. Belhaj and E.H. Saidi, "HyperKahler singularities in superstrings compactification and 2D N=4 Conformal Field Theory"; hep-th/0002205

[11] C. G. Callan, C. Lovelace, C.R. Nappi, S.A. Yost, "String loop corrections to Beta Functions"; Nucl. Phys.B 288(1987) 525.

[12] E. M. Sahraoui; Proceedings of the Workshop on "Noncommutative Geometry, Superstring theories and Particle Physics"; 16-17 June 2000 Rabat Morocco. to appear 
[13] J. Wess, "Lectures at Workshop On Noncommutative Geometry, Superstring theories and Particle Physics"; 16-17 June 2000 Rabat Morocco.

[14] J. Madore, S. Schraml. P.Schupp and J. Wess, "gauge theory on noncommutative spaces"; hep-th/0001203

[15] H. Weyl, "Quantum mechanik und gruppen theorie"; Z. Physik 46 (1927)1

[16] M. Chaichain, A. Demichev and P. Presnajder, "Quantum field theory on Noncommutative spaces times and the persistence of ultraviolet Divergences"; hep-th/981218.

[17] M- Sheikh - Jabbari, "One Loop renormalisability of Supersymmetric Yang -Mills Theories on Noncommutative torus "; JHEP 06(1999) 015.hep-th/9903107.

[18] J. Maldacena and J. Russo, "Large N limit of Non-commutative Gauge theories"; hep-th/9908134.

[19] S. Minwalla, M.V. Raamsdonk and N. Seibeg, "Noncommutative perturbative Dynamics"; hep-th/9912072

[20] S. Cho, R. Hinterding, J. Madore and H. Steinacker, "Finite field theory on noncommutative geometry"; hep-th/9903239.

[21] A. Galperin, E. Ivanov, S. Kalitzin, V. Ogievetsky and E. Sokatchev; Class . Quant. Grav 1(1984) 469.

[22] A. Galperin, Anh ky Nguyen and E. Sokatchev; Class. Quant. Grav 4(1987)1235

[23] A. Galperin, E. Ivanov, V. Ogievetsky and E. Sokatchev; Class. Quant. Grav. 4(1987) 1255

[24] A. Galperin, E. Ivanov, V. Ogievetsky and E. Sokatchev; Com. Math 103(1986)515

[25] E. Sahraoui and E. H. Saidi, " On the completely integrable four dimensions N=2 hypermultiplet self - couplings"; Class . Quant. Grav. 16(1999) 1605.

[26] A. Galperin, E. Ivanov, V. Ogievetsky and P. K. Townsend; Class. Quant. Grav 3 (1986)625

[27] A. Galperin, E. Ivanov, V. Ogievetsky and E. Sokatchev; Annals of physics 185(1988)1

[28] S. Kalitzin and E. Sokatchev; Class. Quant. Grav. 4(1987)173 
[29] A. Galperin, E. Ivanov, V. Ogievetsky and E. Sokatchev; Class. Quant. Grav. $2(1985) 601$

[30] J. A. Bagger, A. Galperin, E. Ivanov and V. Ogievetsky; Nucl. Phys B 303 (1988) 522

[31] T. Lhalabi and E.H. Saidi; Nucl. Phys. B 335 (1990) 689

[32] A. Galperin, E. Ivanov, V. Ogievetsky and E. Sokatchev; Anals of Physics. 185(1988) 22

[33] G. W. Gibbons, D. Olivier, P. J. Ruback and G. Valent; Nucl. Physi. B 296(1988)679

[34] C. N. Yang; Phys Rev. Lett. 38(1977)1377

[35] B. Zumino; Phys. lett. B 87 (1979)203

[36] L. Alvarez Gaumé and D. Z Freedman; Commun. Math. Phys. 80(1981)443

J. Bagger and E. Witten; Nucl. Phys. B 222 (1983)1. 